\title{
Kaposi's sarcoma associated herpesvirus entry into target cells
}

\section{Sayan Chakraborty, Mohanan Valiya Veettil * and Bala Chandran}

H. M. Bligh Cancer Research Laboratories, Department of Microbiology and Immunology, Chicago Medical School, Rosalind Franklin University of Medicine and Science, North Chicago, IL, USA

\section{Edited by:}

Keiji Ueda, Osaka University Graduate

School of Medicine, Japan

\section{Reviewed by:}

Keiji Ueda, Osaka University Graduate School of Medicine, Japan

Frank Neipel, Universitaet Erlangen,

Germany

\section{*Correspondence:}

Mohanan Valiya Veettil, H. M. Bligh

Cancer Research Laboratories,

Department of Microbiology and

Immunology, Chicago Medical

School, Rosalind Franklin University of

Medicine and Science, North

Chicago, IL, USA.

e-mail:mohanan.veettil@

rosalindfranklin.edu
Herpesvirus infection of target cells is a complex process involving multiple host cell surface molecules (receptors) and multiple viral envelope glycoproteins. Kaposi's sarcoma associated herpesvirus (KSHV or HHV-8) infects a variety of in vivo target cells such as endothelial cells, B cells, monocytes, epithelial cells, and keratinocytes. KSHV also infects a diversity of in vitro target cells and establishes in vitro latency in many of these cell types. KSHV interactions with the host cell surface molecules and its mode of entry in the various target cells are critical for the understanding of KSHV pathogenesis. KSHV is the first herpesvirus shown to interact with adherent target cell integrins and this interaction initiates the host cell pre-existing signal pathways that are utilized for successful infection. This chapter discusses the various aspects of the early stage of KSHV infection of target cells, receptors used and issues that need to be clarified, and future directions. The various signaling events triggered by KSHV infection and the potential role of signaling events in the different stages of infection are summarized providing the framework and starting point for further detailed studies essential to fully comprehend the pathogenesis of KSHV.

Keywords: KSHV, entry, tropism, integrins, signaling, endocytosis, receptors

\section{INTRODUCTION}

Viruses are obligatory parasites that critically rely on their ability to transmit their genome from infected to uninfected host organisms. Being an inert particle, viruses have successfully evolved to exploit the behavior and physiology of their host. Viral infection induces the activation of various endogenous responses that enable it to permeate through cell membranes and other barriers to reach the cytoplasm or nucleus.

Herpesviruses have a large double stranded DNA genome enclosed in the viral protein shell (capsid) surrounded by a tegument layer which is enclosed in a lipid envelope with at least eight distinct viral envelope glycoproteins. A characteristic property of these viruses is that after primary infection they establish lifelong latent infection in the infected host with periodic reactivation and re-infection. KSHV or human herpesvirus-8 (HHV-8) is a member of the $\gamma 2$-lymphotropic-oncogenic herpesviruses. KSHV is etiologically associated with Kaposi's sarcoma (KS) and with at least two lymphoproliferative malignancies, primary effusion lymphoma (PEL) and multicentric Castleman's disease (MCD). It is the newest member of the human herpesvirus family and is closely related to $\gamma-1$ Epstein-Barr virus (EBV), $\gamma$-2 herpesvirus saimiri (HVS) and Rhesus monkey rhadinovirus (RRV; Ganem, 2007a,b). KSHV has a double stranded DNA genome of about $\sim 160$-kb encoding more than 90 ORFs designated 4-75 by their homology to HVS ORFs. The genome contains gene blocks conserved with other herpesviruses as well as divergent regions encoding more than 20 KSHV unique genes ( $\mathrm{K}$ genes). KSHV encodes several proteins that are homologs of host proteins with immunomodulatory, anti-apoptotic, signal induction, transcriptional regulation, and other functions (Cesarman et al., 1996; Neipel et al., 1997).

The first step of any viral infection is governed by binding and entry into target cells. Therefore, to control KSHV infection, a detailed understanding of how KSHV infects its target cells utilizing the varied set of cellular receptors, envelope glycoproteins, signaling, and modes of entry is essential. Recent advances indicate that KSHV interacts with multiple host cell surface receptors of adherent target cells and these interactions induce a network of rapid intracellular signaling pathways, which facilitate the various steps of successful infection. Here, we review the important steps involved in KSHV entry into target cells utilizing viral envelope-cellular receptor interactions, and signal cascades inducing dynamic cell membrane changes leading to a productive latent infection.

\section{KSHV TROPISM}

To better understand the underlying multistep complex entry mechanism(s) initiated by KSHV, one must appreciate the broad variety of cell types infected both in vivo and in vitro.

In vivo KSHV has a broad tropism as suggested by the detection of its genome and transcripts in a variety of in vivo cell types such as CD19+ peripheral blood B cells, endothelial cells, monocytes, keratinocytes, and epithelial cells (Ganem, 2007b). Latent KSHV DNA is present in vascular endothelial and spindle cells of KS lesions, associated with expression of latency-associated ORF73 (LANA-1), ORF 72 (v-cyclin D), K13 (v-FLIP), and K12 (Kaposin) genes and microRNAs (Boshoff et al., 1995; Dupin et al., 1999; Ganem, 2007b). Lytic infection is also detected in $<1 \%$ of infiltrating inflammatory monocytic cells of KS lesions (Dourmishev 
et al., 2003; Ganem, 2007b). Available evidences suggest that B cells and monocytes are the major reservoir of in vivo latent infection. Cell lines with B cell characteristics, such as BC-1, BC-3, BCBL-1, HBL-6, and JSC have been established from PEL tumors (Dourmishev et al., 2003; Ganem, 2007b). In PEL cells, in addition to the above set of latent genes, K10.5 (LANA-2) gene is also expressed (Parravicini et al., 2000; Ganem, 2007b). About 1-3\% of PEL cells spontaneously enter lytic cycle and virus induced from these cells by chemicals serve as the source of virus. Multiple genome copies of both KSHV and EBV exist in latent form in BC-1, HBL- 6 , and JSC cells while BCBL- 1 and BC- 3 cells carry only the KSHV genome (Ganem, 2007b). An endothelial cell line carrying KSHV has not been established from KS lesions since KS cells grow poorly in cell culture and viral DNA is lost within a few passages (Ganem, 2007b).

Kaposi's sarcoma associated herpesvirus has been shown in vitro to infect several types of human cells such as B, endothelial, epithelial, fibroblast cells, CD34+ stem cell precursors of dendritic cells (DCs), and monocytes (Ganem, 2007b). KSHV also infects owl monkey kidney cells, baby hamster kidney (BHK-21) cells, Chinese hamster ovary ( $\mathrm{CHO}$ ) cells, and mouse fibroblasts cells (Parravicini et al., 2000; Akula et al., 2001a,b, 2002; Birkmann et al., 2001; Bechtel et al., 2003; Inoue et al., 2003; Garrigues et al., 2008; Jarousse et al., 2008).

Infection of primary B cells by KSHV does not result in immortalization and a lytic KSHV replication is seen in activated B cells. Another characteristic feature of in vitro infection of human microvascular dermal endothelial cells (HMVEC-d), human umbilical vein endothelial cells (HUVEC), human foreskin fibroblasts (HFF), human endothelial cells immortalized by telomerase (TIME), and human endothelial cells (HEK-293), monkey kidney cells (VERO, CV-1), and mouse fibroblasts (Bechtel et al., 2003) by KSHV is the expression of latency-associated genes and the absence of productive lytic replication and thus providing a reasonable model for studying in vitro latency. However, latent infection of KSHV in vitro is not persistent and leads to the loss of viral genome over time (Grundhoff and Ganem, 2004).

Analysis of in vitro KSHV interaction with adherent target cells and quantitation of infection has been hampered by the absence of a lytic replication cycle and hence a plaque assay.
Since in vitro KSHV infection results in the expression of latencyassociated genes, various methods have been devised to assess the different phase(s) of KSHV infection (Parravicini et al., 2000; Table 1).

\section{KSHV BINDING AND ENTRY INTO TARGET CELLS}

Kaposi's sarcoma associated herpesvirus uses multiple envelope glycoproteins to complete the binding and entry processes. KSHV binding to the target cells and identity of the receptors involved in binding and entry were elucidated by using labeled virus binding to the target cells at $4^{\circ} \mathrm{C}$ as well as other methods (Table 1). These studies have demonstrated that KSHV binds and enters a variety of target cells which include human (293, HFF, HeLa, HMVECd, HUVEC, TIME, BCBL-1, BJAB, Raji), monkey (Vero, CV-1), hamster (BHK-21, CHO), and mouse (Du17) cells. This is demonstrated by the detection of viral DNA, limited viral gene expression, and GFP expression (Table 1). Real-time DNA PCR of internalized KSHV DNA demonstrates a rapid internalization of viral DNA in the infected endothelial and HFF cells (Krishnan et al., 2004).

\section{KSHV ENVELOPE GLYCOPROTEINS-MEDIATORS OF BINDING AND ENTRY}

Kaposi's sarcoma associated herpesvirus envelope glycoproteins play critical roles in mediating virus attachment, entry, assembly, and egress of virus. Like other herpesviruses, KSHV encodes five conserved glycoproteins gB (ORF 8), gH (ORF 22), gL (ORF 47), gM (ORF 39), and gN (ORF 53; Cesarman et al., 1996; Neipel et al., 1997; Ganem, 2007a). In addition to these glycoproteins, KSHV also encodes the unique lytic cycle associated glycoproteins ORF 4, gpK8.1A, gpK8.1B, K1, K14, and K15 (Cesarman et al., 1996; Neipel et al., 1997; Ganem, 2007a). Among these, ORF 4, gB, $\mathrm{gH} / \mathrm{gL}, \mathrm{gM} / \mathrm{gN}$, and gpK8.1A are associated with KSHV envelopes (Baghian et al., 2000; Parravicini et al., 2000; Akula et al., 2001a,b; Birkmann et al., 2001; Wang et al., 2001; Naranatt et al., 2002; Koyano et al., 2003).

Kaposi's sarcoma associated herpesvirus $\mathrm{gB}$ is a major envelope glycoprotein. It is synthesized as a $110-\mathrm{kDa}$ precursor protein which undergoes cleavage and processing to yield envelope associated disulfide linked 75 and $54-\mathrm{kDa}$ polypeptides with high mannose and complex sugars (Baghian et al., 2000; Akula et al., 2001a; Wang et al., 2003). KSHV-gB mediates viral binding and

Table 1 | Methods employed to study the various stages of in vitro KSHV infection.

\begin{tabular}{|c|c|}
\hline Stage of infection & Detection methods \\
\hline 1. Binding & $\begin{array}{l}\text { [H3] Thymidine labeled virus and FITC labeled (FACS) binding assay; viral DNA (ORF73 gene) DNA } \\
\text { quantitation by real-time DNA PCR; electron and confocal microscopy. }\end{array}$ \\
\hline 2. Signal induction & $\begin{array}{l}\text { Quantitation of signal molecules induction by Western blots and ELISA; use of chemical inhibitors or } \\
\text { dominant-negative signal molecules. }\end{array}$ \\
\hline 3. Viral DNA internalization (entry) & $\begin{array}{l}\text { Real-time DNA PCR for KSHV ORF73 gene after removal of unbound/partially bound virus by } \\
\text { trypsin-EDTA; electron and confocal microscopy. }\end{array}$ \\
\hline 4. Cytoplasmic trafficking of KSHV & $\begin{array}{l}\text { Confocal microscopy by colocalizing virus with microtubules and endosomal vesicles; physiological } \\
\text { ligand uptake assays. }\end{array}$ \\
\hline 5. Nuclear delivery of KSHV DNA & Real-time DNA PCR for ORF73 gene in the isolated nuclei of infected cells. \\
\hline 6. Viral gene expression, host gene manipulation & $\begin{array}{l}\text { Real-time RNA PCR for KSHV and host gene expression; confocal microscopy and FACS for KSHV } \\
\text { ORF73 gene and GFP expression. }\end{array}$ \\
\hline
\end{tabular}


entry by interacting with cell surface heparan sulfate and integrins $\alpha 3 \beta 1, \alpha \mathrm{V} \beta 3$, and $\alpha \mathrm{V} \beta 5$ (Akula et al., 2001a,b, 2002; Veettil et al., 2008). KSHV-gB interaction with target cells modulates the host cell signaling pathways by inducing integrin associated focal adhesion kinases (FAK), Src, PI3-K, and Rho-GTPase activities (Sharma-Walia et al., 2004). Studies have demonstrated that besides playing a role in KSHV binding and entry of target cells, gB is critical for virus maturation and egress (Krishnan et al., 2005).

Distinct from other herpesviruses, the KSHV gpK8.1 gene encodes two alternatively spliced messages yielding glycoprotein gpK8.1A and gpK8.1B. Both gpK8.1A and gpK8.1B contain $\mathrm{N}$ - and O-linked sugars, and gpK8.1A is the predominant form detected within infected cells and in the virion envelopes (Neipel et al., 1997; Zhu et al., 1999a,b). Like gB, gpK8.1A also possesses the heparan sulfate binding motif and interacts with cell surface heparan sulfate molecules (Wang et al., 2001). Similar to herpes simplex type 1 (HSV-1) gB glycoprotein, recent studies have reported that KSHV $\mathrm{gB}$ and gpK8.1A are enriched in membrane microdomains, lipid rafts (LRs), during early infection in endothelial cells (Bender et al., 2003; Chakraborty et al., 2011).

Like in other $\alpha, \beta$, and $\gamma$-herpesviruses, KSHV gH and $\mathrm{gL}$ form a non-covalently linked complex consisting of $120-\mathrm{kDa} \mathrm{gH}$ and $42-\mathrm{kDa}$ gL proteins. KSHV gL is required for processing and intracellular transport of $\mathrm{gH}$ and this complex is critical for KSHV entry (Naranatt et al., 2002). Recent studies show that KSHV gH, as well as complement binding KSHV ORF4, also interacts with cell surface heparan sulfate (Mark et al., 2006; Hahn et al., 2009). Studies have demonstrated that anti-gH and anti-gL antibodies inhibit KSHV entry without affecting binding to the target cells (Naranatt et al., 2002).

Kaposi's sarcoma associated herpesvirus gM and $\mathrm{gN}$ also form a glycosylated heterodimeric complex and are involved in virus penetration and egress. $\mathrm{gN}$ has been shown to be essential for proper post translational modification and transport of gM to the cell surface (Koyano et al., 2003). KSHV gM and gN forms a heterodimeric complex and were shown to inhibit cell fusion in an in vitro cell fusion assay (Koyano et al., 2003).

\section{CELLULAR RECEPTORS RECOGNIZED BY KSHV}

Most cell surface molecules that herpesviruses bind fall into two main categories depending on the functional consequences of the interaction. The major groups include the attachment or binding factors and entry receptors. Attachment factors promote binding and concentration of viruses on target cells but may not be very specific. Often, these involve charge interactions involving heparan sulfate or other carbohydrate moiety bearing molecules.

The other group comprises entry receptors, including a broad variety of cell surface molecules capable of either viral fusion to cell membranes or initiating signaling to promote endocytosis. Entry receptors are highly specific and vary by cell types. Often, these molecules are internalized along with the virus, hence are actively utilized by viruses.

\section{HEPARAN SULFATE AS KSHV ATTACHMENT FACTOR IN TARGET CELLS}

Like many herpesviruses, KSHV utilizes ubiquitous heparan sulfate (HS) molecule for binding to most of its target cells. HS bears carbohydrate residues that facilitate concentration of virus particles on target cells owing to charge interactions. KSHV infection can be inhibited by soluble heparin but not by chondroitin sulfates A and C (Akula et al., 2001a). Pre-treatment of KSHV with soluble heparin prevents virus binding and subsequent signal induction. This is a specificity control for KSHV-induced phenomena. Several B cell lines and primary B cells lack the Extl enzyme, which promotes glycosylation in HS biosynthesis. Due to this, B cells have low HS expression, and thereby refractory to KSHV infection. However, expression of $\mathrm{HS}$ in $\mathrm{BJAB}$ (EBV negative cell line) results in greater susceptibility to KSHV infection (Jarousse et al., 2008). These studies suggest that KSHV infection of BJAB cells depends on the level of expression of HS; BJAB cells expressing HS were readily infected while cells lacking HS were not infected (Kabir-Salmani et al., 2008).

Kaposi's sarcoma associated herpesvirus envelope glycoproteins gB, gpK8.1A, ORF4, and gH bind to cell surface HS molecules, thereby emphasizing the importance of cell surface HS for KSHV attachment to target cells (Birkmann et al., 2001; Akula et al., 2002; Wang et al., 2003; Hahn et al., 2009). Incubation of KSHV with soluble heparin and enzymatic removal of cell surface HS by heparinase I and III inhibits KSHV infectivity. Binding of the soluble forms of gB and gpK8.1A to target cells is saturable and can be blocked by soluble heparin (Birkmann et al., 2001; Wang et al., 2001, 2003).

Many proteins possess more than one of the two heparin binding domains (HBD), containing the XBBXBX and XBBBXXBX sequences, where $B$ is a positively charged basic amino acid (lysine, arginine, or histidine) flanked by an additional positively charged residue separated by hydrophobic amino acids "X." The extracellular domain of KSHV gB possesses 108HIFKVRRYRK117, which is a BXXBXBBXBB type $\mathrm{HBD}$, and is conserved throughout the $\gamma 2$ herpesviruses. KSHV gpK8.1A also possesses two possible atypical heparin-binding motifs, ${ }^{150}$ SRTTRIRV $^{157}$ (XBXXBXBX) and ${ }^{182}$ TRGRDAHY $^{189}$ (XBXBXXBX) whereas KSHV gH lacks the typical HBD.

Several lines of evidence indicate that KSHV-gB and gpK8.1A bind to cell surface HS molecules (Akula et al., 2001b; Birkmann et al., 2001; Wang et al., 2003). Binding of soluble forms of the proteins made in baculovirus is saturable and can be blocked by soluble heparin (Wang et al., 2001, 2003). Full length gB and gpK8.1A in the virion envelope specifically bind heparin-agarose, and can be eluted by high concentrations of soluble heparin, but not by chondroitin sulfates (Wang et al., 2001; Akula et al., 2002). KSHV-gpK8.1A binds to heparin with an affinity comparable to that of glycoproteins $\mathrm{B}$ and $\mathrm{C}$ of herpes simplex virus and gpK8.1A binds more strongly than gB (Wang et al., 2003).

\section{DC-SIGN AS KSHV ENTRY RECEPTORS}

Dendritic cell specific intercellular adhesion molecule-3 (ICAM3) grabbing non-integrin (DC-SIGN; CD209) is a C-type lectin present on the dermal DC surface. It has been shown to be used by many viruses as a receptor including human immunodeficiency virus (HIV) and more recently by Bunyaviruses (Geijtenbeek et al., 2003; Lozach et al., 2011). Similarly, KSHV also appears to utilize DC-SIGN during infection of human myeloid DCs, macrophages, 
and activated B cells (Rappocciolo et al., 2006, 2008). DC-SIGN blocking monoclonal antibodies and mannan efficiently inhibited KSHV binding and infection. However, the pre-treatment of cells with anti-DC-SIGN antibodies did not completely block KSHV binding and infection possibly due to binding to HS and/or other receptors. KSHV envelope glycoprotein(s) interacting with DC-SIGN is not known yet and KSHV gB with its high mannose sugar is a potential candidate. Recent studies have shown that activated B cells are infected more efficiently due to increased expression of DC-SIGN. However, whether HS and other KSHV receptors are also expressed at higher levels possibly contributing to the increased infectivity in activated B cells need to be studied further.

Recent studies have also elucidated that KSHV effectively binds and enters THP-1, a human acute monocytic leukemic cell line, using heparan sulfate and integrins (Kerur et al., 2010). Blocking DC-SIGN in these cells did not affect KSHV binding; however KSHV entry was reduced, suggesting that KSHV utilized DC-SIGN as part of the entry receptor(s) in addition to the previously identified integrins ( $\alpha 3 \beta 1, \alpha \mathrm{V} \beta 3$, and $\alpha \mathrm{V} \beta 5$ ) in THP-1 cells (Kerur et al., 2010).

\section{KSHV IS THE FIRST HERPESVIRUS SHOWN TO UTILIZE INTEGRINS AS ENTRY RECEPTORS IN ADHERENT CELLS}

Several viruses utilize multiple integrins for target cell infection and engaging integrin receptors leading to induction of potent signaling responses critical for virus infectivity. KSHV-gB possesses an integrin-binding RGD (Arg-Gly-Asp) motif at amino acids 27-29. The RGD motif is the minimal peptide sequence of many integrin ligands known to interact with subsets of cellular integrins. Several studies have demonstrated that $\alpha 3 \beta 1, \alpha \mathrm{V} \beta 3$, and $\alpha \mathrm{V} \beta 5$ integrins play roles in KSHV infection. Using RGD peptides, antibodies against RGD-gB (RGDTFQTSSSPTPPGSSS), and the extracellular matrix (ECM) protein fibronectin studies have shown the role of integrins in KSHV infection of HMVEC-d and HFF cells (Akula et al., 2002). In HMVEC-d and HFF cells, KSHV interacts with integrin $\alpha 3 \beta 1$ as demonstrated by a $30-50 \%$ reduction in infection by pre-treating cells with function blocking anti- $\alpha 3$ and $\beta 1$ antibodies and by mixing virus with soluble $\alpha 3 \beta 1$ integrin before infection, as well as the immunoprecipitation of virus- $\alpha 3$ and $\beta 1$ complexes by anti-KSHV-gB antibodies (Akula et al., 2002). Though expression of $\alpha 3$ integrin in $\mathrm{CHO}$ cells increases infectivity, the levels of infection do not reach that observed in the HMVEC-d and HFF cells strongly suggesting that KSHV uses multiple receptors (Akula et al., 2002; Naranatt et al., 2003; Krishnan et al., 2004).

Virus binding and DNA internalization studies suggest that integrins $\alpha \mathrm{V} \beta 3$ and $\alpha \mathrm{V} \beta 5$ also play roles in KSHV entry (Veettil et al., 2008). Variable levels of inhibition of virus entry into adherent HMVEC-d, 293 and Vero cells, and HFF was observed by pre-incubating virus with soluble $\alpha \mathrm{V} \beta 3, \alpha \mathrm{V} \beta 5$, and $\alpha 3 \beta 1$ integrins, and cumulative inhibition was observed with a combination of integrins. Confocal microscopy studies confirmed the association of KSHV with $\alpha 3 \beta 1$ integrins (Veettil et al., 2008; Chakraborty et al., 2011). A study also revealed the roles of integrins ( $\alpha 3 \beta 1$, $\alpha \mathrm{V} \beta 3$, and $\alpha \mathrm{V} \beta 5$ ) in the entry of KSHV in THP-1 and primary monocyte cells (Kerur et al., 2010).

\section{DISCREPANCIES REGARDING THE ROLE OF INTEGRINS IN KSHV BIOLOGY}

Two studies did not detect a role for $\alpha 3 \beta 1$ in KSHV infection. However, methodological differences could explain the discrepancies. For example, Inoue et al. (2003) reported the inability of soluble $\alpha 3 \beta 1$ integrin and RGD peptides to block KSHV infectivity in the 293-T cell line. However, the validity of this observation is questionable since in this study, cells were pre-treated with RGD peptide and infected with KSHV by using centrifugation and polybrene. Both centrifugation and polybrene are known to enhance virus infection without the need for the virus to interact with specific receptors. It is well known that polybrene forming a complex with viral envelope is used for gene delivery into various target cells bypassing the need of specific receptors. Moreover, Inoue et al. (2003) studies also pre-incubated the cells with integrins, washed, and infected with KSHV. This is not a correct design of experiment since to demonstrate the role of integrin in any viral infection cells need to be incubated first with anti-integrins antibodies, but not soluble integrins, prior to viral infections. Similarly, virus needs to be pre-incubated with soluble integrins before addition to cells to demonstrate the role of respective integrin in viral infection.

Another study utilized a 15-mer-AHSRGDTFQTSSGCG peptide of KSHV-gB and demonstrated that this peptide mediated the human fibrosarcoma HT1080 cell adhesion which was blocked by $\alpha \mathrm{V} \beta 3$ and $\alpha \mathrm{V} \beta 5$ antibodies while peptide bound beads detected only $\alpha$ V $\beta 3$ integrin (Garrigues et al., 2008). The GCG amino acids in the peptide used in this study are not present in the KSHV-gB sequence and may potentially give rise to dimers and multimers due to the cystine residue (Garrigues et al., 2008). Though HT1080 cell infection was inhibited by anti- $\alpha \mathrm{V} \beta 3$ antibodies, the ability of anti- $\alpha 3 \beta 1$ and $\alpha \mathrm{V} \beta 5$ antibodies to block infection was not examined (Garrigues et al., 2008). Moreover, co-immunoprecipitation and colocalization studies to demonstrate the direct association of $\mathrm{KSHV}$ with integrins were not done in these studies. Hence, to disproof that KSHV infection depends upon $\alpha 3 \beta 1$, studies should be carried out with the same target cells that have been used to show the role of $\alpha 3 \beta 1$ integrin in KSHV target cell infection. Moreover, the differential ability of integrins to block infection remains questionable in HT1080 cells since another study could not infect these cells (Veettil et al., 2008). Mouse keratinocytes lacking $\alpha 3 \beta 1$ were infectable with KSHV and expression of human $\alpha 3$ resulted in only $55 \%$ of infection in these cells. Even though the level of $\alpha \mathrm{V} \beta 3$ in these cells and the ability of anti- $\alpha \mathrm{V} \beta 3$ to block KSHV infection were not tested, it was concluded that $\alpha 3 \beta 1$ expression must have a dominant-negative effect on $\alpha \mathrm{V} \beta 3$ integrin. Later studies clearly demonstrating the association of KSHV with $\alpha 3 \beta 1$ integrin and LR-KSHV and $\alpha 3 \beta 1$ integrins (Veettil et al., 2008; Chakraborty et al., 2011), it is clear the conclusions from the above two studies is not correct and should not be considered further.

The discrepancies in the usage of different integrins were expected since it is common for different cells to express different combinations of integrins and a specific integrin could be one of the receptors in some but not in all target cells of KSHV. Since herpesvirus-cell receptor interactions are temporarily coordinated events mediated by interactions of viral glycoproteins with one receptor leading to conformational changes in the viral glycoproteins allowing interaction with the next receptor(s), 
detection of $\alpha 3$ and $\beta 1$ in HFF or HMVEC-d cells incubated with virus or purified gB (Akula et al., 2002) could be representing the event occurring during virus-host cell interactions under physiological conditions. Further studies are required to determine the role of additional receptor(s) in different target cells of KSHV.

Later studies demonstrated that $\alpha \mathrm{V} \beta 3$ and $\alpha \mathrm{V} \beta 5$ integrins also play roles in KSHV entry of adherent target cells (Veettil et al., 2008; Chakraborty et al., 2011; Table 2; Figure 1). Studies have also demonstrated the neutralization of KSHV infection in HMVEC-d and HFF cells upon blocking with anti- $\alpha \mathrm{V} \beta 3$ and $\alpha \mathrm{V} \beta 5$ antibodies. Supporting these findings, KSHV entry was inhibited by soluble $\alpha 3 \beta 1, \alpha V \beta 3$, and $\alpha V \beta 5$ integrins in HMVEC-d, HFF, 293, and Vero cells (Veettil et al., 2008). Though a cumulative inhibition was observed with combinations of integrins, complete block in infection was not achieved which may be due to KSHV's first interaction with HS. The role of integrin in KSHV infection of HUVEC cells, B cells, monocytes, keratinocytes, and other cells has not been studied (Table 2).

\section{ROLE OF xCT AS A KSHV ENTRY RECEPTOR}

Kaleeba and Berger (2006) identified the 12-transmembrane glutamate/cysteine exchange transporter protein $\mathrm{xCT}$ as a fusion-entry receptor in adherent cells. Ectopic expression of xCT rendered the non-susceptible adherent target cells to become susceptible to KSHV infection (Kaleeba and Berger, 2006). xCT is part of the cell surface $125-\mathrm{kDa}$ disulfide linked heterodimeric membrane glycoprotein CD98 (4F2 antigen) complex containing a common glycosylated heavy chain $(80-\mathrm{kDa})$ and a group of $45 \mathrm{kDa}$ light chains. The xCT molecule is one of the light chains (Fenczik et al., 2001; Feral et al., 2007; Kabir-Salmani et al., 2008; Veettil et al., 2008). CD98 was initially identified as a molecule associated with integrin $\alpha 3$ and plays multiple roles including amino acid transport, cell adhesion, fusion, proliferation, and integrin activation. It is interesting to note that CD98 and integrin $\alpha 3$ were identified as fusion regulation protein 1 (FRP-1) and FRP-2, respectively, as this interaction was shown to play crucial roles in cell-cell fusion and virus-induced cell fusion (Fenczik et al., 2001; Feral et al., 2007; Kabir-Salmani et al., 2008; Veettil et al., 2008). Studies show that $\mathrm{xCT}$ is a component of a multimolecular signaling complex formed during KSHV macropinocytosis in HMVEC-d cells (Veettil et al., 2008; Chakraborty et al., 2011). This suggests that xCT plays a role within a multimolecular complex that may help to regulate the signaling pathways associated with the endocytic pathway of the virus. It is possible that the xCT present in the multimolecular complex may initiate a distinct cellular signaling pathway to control the various events associated with KSHV infection. Since a direct interaction of KSHV envelope glycoproteins with $\mathrm{xCT}$ has not been shown as yet, the exact role of XCT in the multimolecular complex and its role in KSHV infection need to be investigated further.

\section{MULTIMOLECULAR INTEGRIN COMPLEX FORMATION DURING EARLY KSHV INFECTION}

CD98 also mediates membrane clustering, $\beta 1$ integrin-mediated signaling events, and stimulation of $\alpha 3 \beta 1$-dependent adhesion of cells and signal transduction cascade of $\alpha \mathrm{V} \beta 3$ integrin (Fenczik et al., 2001; Feral et al., 2007; Kabir-Salmani et al., 2008; Veettil et al., 2008). Co-immunoprecipitation and immunofluorescence studies in KSHV infected HMVEC-d cells have shown a timedependent interaction of CD98/xCT with integrins $\alpha 3 \beta 1, \alpha \mathrm{V} \beta 5$, and $\alpha$ V $\beta 3$ (Veettil et al., 2008). Three different time-dependent temporal patterns of association and dissociation of KSHV interactions with cell surface molecules were observed. Integrin $\alpha \mathrm{V} \beta 5$ interaction with CD98/xCT predominantly occurred by $1 \mathrm{~min}$ post-infection (p.i.) and dissociated at $10 \mathrm{~min}$ p.i., whereas $\alpha 3 \beta 1-$ CD98/xCT interaction was maximal at $10 \mathrm{~min}$ p.i. and dissociated at $30 \mathrm{~min}$ p.i., and $\alpha \mathrm{V} \beta 3-\mathrm{CD} 98 / \mathrm{xCT}$ interaction was maximal at $10 \mathrm{~min}$ p.i. and remained at the observed $30 \mathrm{~min}$ p.i. Confocal microscopy studies confirmed the association of CD98/xCT with $\alpha 3 \beta 1$ and KSHV. Studies also showed that $\alpha 3 \beta 1-\mathrm{CD} 98 / \mathrm{xCT}$ interactions could be inhibited by pre-incubating KSHV with soluble heparin and $\alpha 3 \beta 1$ strongly suggesting that KSHV's first contact occurs with HS, and integrins are essential elements in subsequent CD98-xCT interactions (Veettil et al., 2008). These studies demonstrated temporal interactions of KSHV with a family of functionally related proteins such as HS, integrins, and CD98$\mathrm{xCT}$ molecules in endothelial, epithelial, and fibroblast cells (Wang et al., 2001, 2003; Akula et al., 2002; Veettil et al., 2008).

\section{BIOLOGICAL RELEVANCE OF UTILIZING MULTIPLE ENTRY RECEPTORS BY KSHV}

It is fascinating to note that KSHV uses multiple molecules to enter target cells. The presence of multiple receptors is evolutionarily advantageous for KSHV and one of the major reasons for the broad tropism of the virus. Although virus-receptor interactions are highly specific, the affinity of interactions are low (Marsh

Table 2 | Binding and entry receptors and entry pathways of KSHV in various in vitro human target cells.

\begin{tabular}{|c|c|c|}
\hline Target cells & Receptors recognized & Entry pathw \\
\hline Human foreskin fibroblast cells (HFF) & Heparan sulfate (binding), $\alpha 3 \beta 1, \alpha \vee \beta 3, \alpha \vee \beta 5, x C T / C D 98$ & Clathrin-med \\
\hline Human microvascular dermal endothelial cells (HMVEC-d) & Heparan sulfate (binding), $\alpha 3 \beta 1, \alpha \vee \beta 3, \alpha \vee \beta 5, x C T / C D 98$ & Macropinocy \\
\hline Human umbilical vein endothelial cells (HUVEC) & Heparan sulfate (binding) & $\begin{array}{l}\text { Macropinocy } \\
\text { endocytosis }\end{array}$ \\
\hline $\begin{array}{l}\text { Human embryonic kidney epithelial cells (HEK-293 with } \\
\text { adenovirus } 5 \text { DNA nts 1-4344 integrated into chromosome } \\
19 \text { (19q13.2) }\end{array}$ & Heparan sulfate (binding) & Endocytosis \\
\hline Monocytes & HS, DC-SIGN & Endocytosis \\
\hline B cells & HS, DC-SIGN & Endocytosis \\
\hline
\end{tabular}




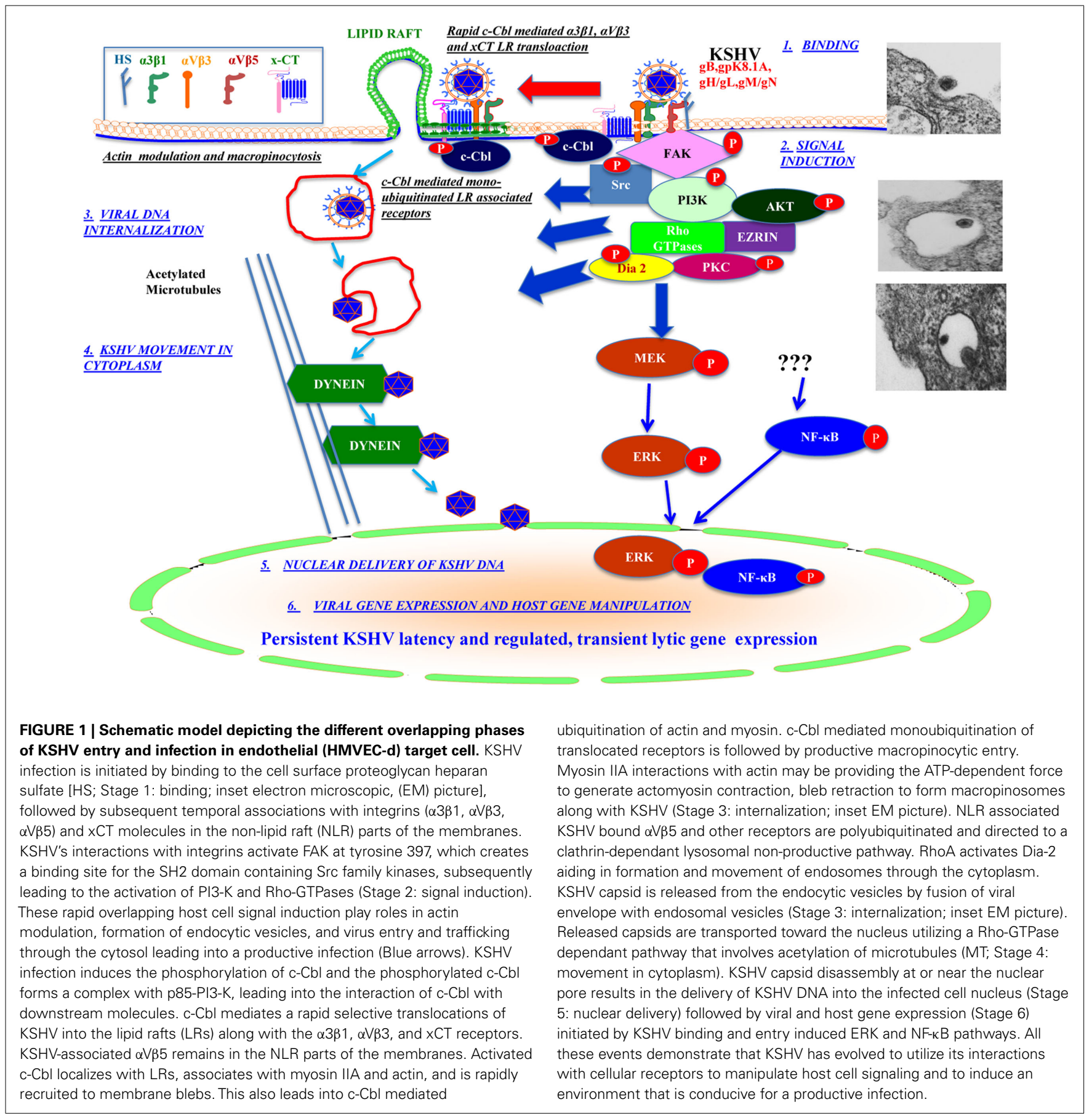

and Helenius, 2006). Hence, KSHV binding to multiple receptors (mainly integrins) possibly increases binding avidity that leads to receptor clustering, a key step to activate signaling pathways. It is widely known that integrins are a signaling hub for a variety of processes including adhesion, motility, and endocytosis.

Deciphering the interactions between entry receptors and signaling leading to active endocytosis of viruses has been an active area of research for the past decade. However, in KSHV biology, recent advancements have revealed that receptor recruitment by KSHV is tightly succeeded by an active modulation of host cell membranes involving actin-myosin and other cellular signaling (Raghu et al., 2009; Valiya Veettil et al., 2010; Chakraborty et al., 2011). Detailed understanding of how KSHV induced such changes in the cell membrane very early during infection comes from its ability to manipulate a variety of host cell molecules to regulate signaling. The usage of multiple receptors possibly adds greater gradient to the succeeding signaling events required for efficient entry into target cells. 


\section{KSHV-INDUCED SIGNALING DURING EARLY INFECTION}

Many viruses, including KSHV make use of host cell signaling to enter target cells and establish productive infection. Binding and interaction of KSHV glycoproteins with integrins and other cellular receptors initiates intracellular signaling cascades to induce internalization. This internalization appears to be very rapid, reaching a peak by 60 -min p.i. (Krishnan et al., 2004) and therefore signaling induced by the virus is very active within this time frame. Integrins are closely associated with FAK and Src kinases and is known to regulate them through a variety of other pathways. Since KSHV utilized a variety of integrins as its cellular receptor, it is evident that the virus has evolved ways to induce the integrin associated signaling.

Kaposi's sarcoma associated herpesvirus induces the phosphorylation of FAK and the subsequent phosphorylation of a variety of focal adhesion associated signal molecules such as Src, PI3$\mathrm{K}$, Rho-GTPases (RhoA, Rac, and Cdc42), and diaphanous-2, as well as several other downstream effector molecules, producing actin rearrangements that eventually lead to the internalization of KSHV. Induction of these signaling pathways is critical for active internalization of KSHV in target cells. The fact that a low multiplicity of infection is able to induce a sequential cascade of signaling events is a unique feature of KSHV infection. Several studies have elucidated that a low dose of KSHV (5-10 DNA copies/cell) is able to induce signaling events critical for entry, expression of viral genes, and latent infection (SharmaWalia et al., 2005; Veettil et al., 2008; Valiya Veettil et al., 2010; Chakraborty et al., 2011). KSHV induces macropinocytic blebs as early as $1 \mathrm{~min}$ p.i., engage clustering of integrins, activate adaptors like c-Cbl, mediate receptor translocations, and induce ERK1/2 for establishing latent infection (Sharma-Walia et al., 2005; Veettil et al., 2008; Valiya Veettil et al., 2010; Chakraborty et al., 2011).

\section{INTEGRIN ASSOCIATED SIGNALING: FOCAL ADHESION KINASES AND THEIR RELEVANCE IN KSHV ENTRY}

Focal adhesions are multifunctional organelles that mediate cell-ECM adhesion, force transmission, cytoskeletal regulation, and signaling. Focal adhesions consist of a complex network of trans-plasma-membrane integrins and cytoplasmic linking of the ECM to the actin cytoskeleton (Riveline et al., 2001). FAK, a major component of focal adhesions, is a multidomain non-receptor tyrosine kinase involved in signaling downstream of integrins. Ligand interaction with integrins activates FAK by autophosphorylation at tyrosine 397, a key step initiating integrin outside-in signaling (Calderwood et al., 2000; Giancotti, 2000). Activated FAK associates with a lot of other signal molecules to regulate a broad range of functions including cell growth, endocytosis, and apoptosis (Giancotti, 2000, 2003).

Kaposi's sarcoma associated herpesvirus infection induced tyrosine phosphorylation of FAK within minutes of infection in HMVEC-d, HFF, 293, and FAK +/+ mouse Dul7 fibroblasts (Akula et al., 2002; Naranatt et al., 2003; Wang et al., 2003; Sharma-Walia et al., 2004, 2005; Veettil et al., 2006; Raghu et al., 2007). Soluble glycoprotein gB also induced FAK autophosphorylation (Wang et al., 2003; Sharma-Walia et al., 2004) and the phosphorylated FAK colocalizes with Src, RhoA, and cytoskeletal proteins like vinculin and paxillin in the infected cells (Akula et al., 2002; Wang et al., 2003; Sharma-Walia et al., 2004; Veettil et al., 2006). Additionally, virus or gB pre-incubated with soluble $\alpha 3 \beta 1$ integrin or a soluble form of $\mathrm{gB}$ in which the RGD sequence had been mutated inhibited the activation of FAK (Akula et al., 2002; Wang et al., 2003; Sharma-Walia et al., 2004). Since FAK activation is a hallmark of integrin-mediated signaling, FAK knockout cells or cells transfected with integrin have been used to study the role of FAK in integrin-mediated signaling. KSHV infection studies with FAK negative (Du3) and FAK positive (Du17) mouse fibroblasts and $\mathrm{CHO}$ cells transfected with human $\alpha 3$ integrin demonstrated a significant role of FAK in KSHV infection (Naranatt et al., 2003; Krishnan et al., 2006). Heparin inhibited the binding of KSHV in both DU3 and DU17 cell types. FAK negative Du3 cells showed an approximately $70 \%$ reduction in KSHV DNA internalization and over-expression of FAK (Du17) increased viral DNA internalization, thus suggesting that FAK plays a significant role in signaling and KSHV entry. In addition to this, over-expression of FAK-related non-kinase (FRNK), a FAK dominant-negative inhibitor, significantly decreased KSHV entry in DU17 cells. Decreased viral entry, nuclear delivery, and viral gene expression in Du3 cells suggest that another protein may be able to compensate for FAK's function early during infection. KSHV infection in Du3 cells induced the phosphorylation of the FAK-related proline-rich tyrosine kinase (Pyk2) molecule, which demonstrated that the Pyk2 molecule compensate for the loss of some of the functions of FAK in FAK negative cells during KSHV infection. Moreover, inhibition of Pyk2 by an autophosphorylation mutant of Pyk2 also significantly reduced viral entry in DU3 cells (Krishnan et al., 2006). Since activation of FAK plays a central role in integrin-mediated signaling, rearrangement of actin and endocytosis, KSHV must have evolved to take advantage of these signaling pathways both to promote entry and the subsequent steps of infection (Figure 1).

\section{ROLE OF SrC AND PI3-KINASES IN KSHV ENTRY AND INFECTION}

The autophosphorylation site of FAK (Tyr397) creates a binding site for the SH2 domain of Src kinases and the p85 subunit of PI3K. KSHV infection induced a strong phosphorylation of Src within minutes of infection, and the phosphorylated Src colocalized with FAK (Veettil et al., 2006). KSHV-gB also induced the FAK dependent Src phosphorylation in adherent target cells (Sharma-Walia et al., 2004) and the activated Src kinases then phosphorylate a number of FA components. Furthermore, Src is required for the activation of PI3-K and other downstream targets such as RhoGTPases. The critical role of Src in the KSHV entry process has been validated by multiple lines of evidences such as the failure of KSHV to enter Src negative mouse fibroblast cells (unpublished observation), increase in Src activity by LR disruption resulting in enhanced virus entry (Raghu et al., 2007). Another observation showed that RhoA-GTPase facilitated KSHV entry into adherent target cells in a Src-dependent manner. This study suggests that KSHV-induced Src is involved in RhoA activation, which in turn results in positive feedback activation of Src to increase viral entry. 
PI3-Kinases (PI3-K) are heterodimeric proteins, consisting of a p85 regulatory subunit and a catalytic p110 subunit while phosphorylation of specific tyrosine residues on the p85 subunit is an indication of PI3-K activation. PI3-kinases play crucial roles in several signaling pathways and regulate multiple functions such as Rho-GTPase activation, apoptosis, survival, and migration (Giancotti, 2000; Sastry and Burridge, 2000). KSHV induces PI3-K within $5 \mathrm{~min}$ p.i. which decreased after $15 \mathrm{~min}$ and this response can be inhibited either by pre-incubating virus with integrin or by the PI3-K inhibitors wortmannin and LY294002 (Naranatt et al., 2003). LY294002 and wortmannin did not affect KSHVgB induced Src phosphorylation whereas the Src kinase inhibitor SU6656 completely blocked KSHV-gB induced p85-PI3-K phosphorylation suggesting that PI3-K is downstream of Src (SharmaWalia et al., 2004). In FAK positive Du17 cells, KSHV-gB induced PI3-K p85 phosphorylation whereas in FAK-null Du3 cells there was no significant induction of PI3-K p85 phosphorylation. This study suggests that FAK is an essential molecule for the induction of PI3-K during KSHV infection. Treatment of cells with PI3-K inhibitors wortmannin and LY294002 reduced PI3-K activation and viral entry in a concentration dependent manner suggesting the role of PI3-K activation in the entry of KSHV in target cells. Induction of PI3-K eventually leads to the induction of RhoGTPases and their effectors (Sharma-Walia et al., 2004; Veettil et al., 2006) which in turn regulates the remodeling of actin, endosome formation, and the movement of endocytic vesicles. Therefore, these studies using chemical inhibitors, dominant-negative proteins, or cells lacking these molecules have demonstrated that FAK, $\mathrm{Src}$, and PI3-K activation were necessary for KSHV entry.

\section{ACTIVATION OF RhOA AND ACTIN DYNAMICS DURING KSHV INFECTION}

RhoA, Rac, and Cdc42-Rho-GTPases are master regulators of a diverse set of signaling pathways, including cytoskeleton rearrangement and morphological changes (Giancotti, 2000; Hall and Nobes, 2000; Ishizaki et al., 2001; Palazzo et al., 2001). Immediately following infection, KSHV induces PI3-K Rho-GTPasedependent cytoskeletal rearrangements and the formation of structures such as filopodia (Cdc42), lamellipodia (Rac), and stress fibers (RhoA) in the target cells (Naranatt et al., 2003; Veettil et al., 2006; Greene and Gao, 2009; Raghu et al., 2009). Soluble $\mathrm{gB}$ induced the FAK-Src-PI3-K Rho-GTPase signaling pathway and extensive cytoskeletal rearrangement in target cells (SharmaWalia et al., 2004). KSHV-induced RhoA colocalized with Src in the infected cells (Sharma-Walia et al., 2004). Ezrin, an actin cross linking protein with the plasma membrane, was also induced by KSHV via Rho-GTPases, thereby modulating membrane changes (Sharma-Walia et al., 2004). Treatment of target cells with a potent RhoA inhibitor, Clostridium difficile toxin $\mathrm{B}(\mathrm{CdTxB})$, or transfecting dominant-negative constructs of RhoA resulted in significant inhibition of KSHV entry by modulation of Src activity (Veettil et al., 2006).

Several studies have demonstrated that RhoA-GTPases mediate rearrangement of cytoskeleton through the activation of its downstream effector molecules, formin family diaphanous 1 and 2 (Hall and Nobes, 2000; Ishizaki et al., 2001; Palazzo et al., 2001). KSHV infection induced diaphenous- 2 without any significant activation of Rac-1 and Cdc42-dependent PAK1/2 and stathmin molecules. Dia-2 co-immunoprecipitated and colocalized with activated Src in the infected cells which were inhibited by Src inhibitors (Veettil et al., 2006). Together with the reduced virus entry in RhoA dominant-negative cells, these results suggest that activated RhoAdependent Dia-2 probably functions as a link between RhoA and Src in KSHV infected cells, mediates the sustained Src activation, and that KSHV-induced Src and RhoA play roles in facilitating entry and nuclear delivery of viral DNA.

\section{KSHV-INDUCED ADAPTOR MOLECULE C-CbI AND ASSOCIATED MEMBRANE DYNAMICS}

The Cbl family of adaptor proteins plays important roles in signal transduction as negative regulators by mediating the ubiquitination and down-regulation of proteins while it acting as a positive regulator through their scaffold function in assembling signaling complexes (Thien and Langdon, 2001; Schmidt and Dikic, 2005). Recent evidences have elucidated that KSHV-induced c-Cbl tyrosine phosphorylation is required for membrane ruffling known as blebs (Valiya Veettil et al., 2010). Blebs are protrusions from the plasma membrane that have often been characterized as a preceding step in macropinocytosis and are known to be induced by many viruses including vaccinia virus and KSHV early during infection (Mercer and Helenius, 2008; Raghu et al., 2009; Valiya Veettil et al., 2010). The role of c-Cbl in the entry and signaling of any viral infection has not been reported. Hence it opens up new directions of studies on the broad cellular requirements required by viruses for infecting target cells.

Kaposi's sarcoma associated herpesvirus is known to induce a variety of endothelial cell membrane alterations including filopodia and bleb formation (Raghu et al., 2009; Valiya Veettil et al., 2010). These studies elegantly deciphered the mechanism behind KSHV-induced membrane bleb formation. KSHV infection increased c-Cbl interaction with PI3-K in a time dependant manner. In KSHV infected cells, activated c-Cbl is recruited to the macropinocytic blebs and associated with its novel interacting partner, myosin IIA inside the membrane blebs very early by 5 min p.i. Studies using shRNA against $\mathrm{c}-\mathrm{Cbl}$ not only reduced the macropinocytic blebs induced by KSHV, but also significantly reduced viral entry by $>70 \%$ and hence KSHV gene expression by $90 \%$ (Valiya Veettil et al., 2010). This study demonstrates that recruitment of c-Cbl-myosin in macropinocytic blebs very early during $\mathrm{KSHV}$ infection is essential for its entry via macropinocytosis in endothelial cells. Although many viruses utilize macropinocytosis to enter target cells, the mechanisms behind it were unclear (Mercer and Helenius, 2008); however, the role $\mathrm{c}-\mathrm{Cbl}$ and myosin IIA in macropinocytic modulation of KSHV potentiate new avenues of interest in the phenomena of membrane dynamicity induced by viruses.

\section{ROLE OF LIPID RAFTS: A SIGNALING PLATFORM UTILIZED BY KSHV IN ENDOTHELIAL CELLS}

Lipid rafts, the detergent resistant microdomains in the exoplasmic leaflet of plasma membranes, are made up of cholesterol and sphingolipids (sphingomyelin and glyco-sphingolipids) and play roles in clustering cell surface receptors and signal molecules (Simmons, 2001). Lipid microdomains favor specific 
protein-protein interactions including ligand receptor interactions activating signal cascades. KSHV binding was not affected upon disruption of LRs by methyl beta cyclodextrin or nystatin, but pre-treatment of HMVEC-d with these LR disrupting drugs significantly reduced the expression of ORF73 and ORF50 (Raghu et al., 2007). Although internalization of viral DNA was increased, the association of internalized viral capsids with microtubules was reduced upon LR disruption, microtubules disorganized, and nuclei associated viral DNA decreased (Raghu et al., 2007).

Mechanistic studies on the role of LRs in KSHV infection revealed more intricate details with relation to entry receptors and signal molecules. A recent study reported that very early during infection ( $1 \mathrm{~min}$ p.i.), $\mathrm{c}$-Cbl induced the selective translocation of KSHV into LRs along with $\alpha 3 \beta 1, \alpha \mathrm{V} \beta 3$, and $\mathrm{xCT}$ receptors, but not $\alpha \mathrm{V} \beta 5$ (Chakraborty et al., 2011). Evidence also suggests that activated $\mathrm{c}$-Cbl localized to LRs at the junctional base of macropinocytic blebs, thereby aiding macropinocytosis (Chakraborty et al., 2011). Such partitioning of entry receptors in LRs by specific signaling adaptors was not reported before and evokes new insights to the molecular mechanisms of viral-host cell interactions, often favoring viral internalization.

The role of LRs is complex and affects KSHV-induced signaling. For instance Raghu et al. (2007) reported that LR disruption affects signal pathways induced by KSHV such that phospho-Src levels had increased without affecting FAK or ERK1/2. However, KSHV-induced PI3-K, Rho-GTPases, and NF- $\kappa$ B activation were significantly reduced. The fact that $\mathrm{p}$-Src increased with LR disruption is indicative of a strong regulation of Src by LRs. LR disruption also affects PI3-K and RhoA with subsequent reduction in KSHV-induced RhoA mediated acetylation and aggregation of MTs (Raghu et al., 2007).

Cellular signaling can be generated in many ways. Viruses most often activate cellular signaling directly by using receptors or induce signaling by clustering specific cell-surface proteins or lipids (Marsh and Helenius, 2006). KSHV serves an excellent model system in this regard as it utilizes both pathways; firstly by activating associated integrin signaling and secondly by clustering activated integrins and signal molecules in LRs, generating significant amplification of the signaling response; however, the molecular partners behind such signaling amplification are yet to be studied.

\section{RECEPTOR UBIOUITINATION: A CRITICAL STEP REQUIRED FOR PRODUCTIVE KSHV INTERNALIZATION}

Ubiquitination of receptors has been recognized as an internalization signal based on the nature and type of ubiquitin modifications (Levkowitz et al., 1998; Dupin et al., 1999). Studies have revealed an important role of c-Cbl, an E3-ubiquitin ligase, in differential ubiquitination of KSHV integrin receptors (Chakraborty et al., 2011). Essentially, LR translocated integrins ( $\alpha 3 \beta 1$ and $\alpha V \beta 3)$ were monoubiquitinated leading to productive macropinocytic entry, whereas non-LR associated $\alpha \mathrm{V} \beta 5$ was polyubiquitinated leading to clathrin mediated entry that was targeted to lysosomes, the non-infectious pathway (Chakraborty et al., 2011). This elucidates the complexities of viral endocytic mechanisms and the ability of KSHV to utilize E3-ubiquitin ligases to regulate and sort out productive pathways.

\section{DIVERSE INTERNALIZATION PATHWAYS OF KSHV IN TARGET} CELLS

Current evidences show that KSHV enters human B cells (Rappocciolo et al., 2008), fibroblast (Akula et al., 2003), epithelial (Inoue et al., 2003; Liao et al., 2003), and endothelial cells (Raghu et al., 2009) by endocytosis. KSHV was detected by electron microscopy in large endocytic vesicles within $5 \mathrm{~min}$ of HMVEC-d and HFF cell infection while fusion of virion envelope with the endocytic vesicles was also observed (Akula et al., 2001a, 2003). Viral capsids were detected in the vicinity of the nuclear membrane by $15 \mathrm{~min}$ p.i. and anti-KSHV-gB and gpK8.1A antibodies colocalized with virus-containing endocytic vesicles (Akula et al., 2001a; Greene and Gao, 2009; Raghu et al., 2009; Table 2).

The roles of different endocytic pathways have been studied using specific inhibitors of each type. Clathrin-mediated endocytosis is the predominant pathway of entry in HFF cells. Chlorpromazine, an inhibitor of clathrin-mediated endocytosis, significantly inhibited entry in HFF cells, whereas nystatin, an inhibitor of caveolae and cholera toxin B, a LR inhibiting agent did not have any effect on entry. A significant inhibition of gene expression was also observed after blocking endosomal acidification by $\mathrm{NH}_{4} \mathrm{Cl}$ and bafilomycin A in HFF cells (Akula et al., 2003; Raghu et al., 2009) suggesting that post internalization endosomal acidification was required for KSHV trafficking. In addition to this, electron microscopy revealed KSHV virions in large endocytic vesicles within 5-min of HFF cell infection and fusion of virion envelope with endocytic vesicles was also observed (Akula et al., 2003; Raghu et al., 2009). Evidence for clathrin-mediated endocytosis has also been observed in BJAB and 293 cells (Akula et al., 2001a; Inoue et al., 2003). KSHV enters THP-1 cells and primary monocytes by clathrin and caveolin dependant endocytosis, which required endosomal acidification (Kerur et al., 2010).

In HMVEC-d and HUVEC cells, entry and gene expression of KSHV were significantly blocked by macropinocytosis inhibitors EIPA and rottlerin. Macropinocytosis of KSHV is an actin dependant endocytic pathway and it was also inhibited by cytochalasin D (Raghu et al., 2009). Cytochalasin D inhibited actin polymerization and formation of lamellipodial extensions significantly inhibited the entry and expression of KSHV (Naranatt et al., 2003; Greene and Gao, 2009; Raghu et al., 2009). The LR inhibiting agents reduced viral gene expression in HMVEC-d cells but not in HUVEC or HFF cells, indicating the role of LRs in KSHV infection (Greene and Gao, 2009; Raghu et al., 2009). Colocalization studies using clathrin-mediated endocytosis marker, transferrin and macropinocytosis marker, dextran showed significant association of KSHV with dextran but not with transferrin or the caveolar marker caveolin. The dynamin inhibitor, dynasore, did not affect viral entry into endothelial cells while inhibiting entry into HFF cells (Raghu et al., 2009). The small GTPase Rab34, a key regulator of macropinocytosis, associated with KSHV and Rab34-siRNA considerably decreased KSHV gene expression (Raghu et al., 2009). These studies suggested that KSHV utilizes the actin polymerization-dependent, dynamin-independent macropinocytic pathway involving a Rab34 GTPase-dependent late endosome and low-pH environment for its infectious entry into HMVEC-d and HUVEC cells (Table 2). 
Recent studies have confirmed the role of LRs in aiding the macropinocytosis of KSHV bound receptors in endothelial cells, whereas non-LR bound receptors were targeted toward a clathrin mediated non-infectious lysosomal pathways (Chakraborty et al., 2011). This study suggests that both macropinocytosis and clathrin-mediated endocytosis are occurring in endothelial cells, however the former being productive while the latter is degradative. KSHV internalized by clathrin-mediated endocytosis clearly associated with lysosomal compartments (Chakraborty et al., 2011). Another report suggested that clathrin-mediated endocytosis is the predominant pathway of entry in endothelial cells. This discrepancy could be due to optimal concentration of the inhibitor used and the method of quantification used to analyze the entry of KSHV (Greene and Gao, 2009). The detection of KSHV by ORF65 (KSHV capsid protein) in perinuclear regions of HUVECs does not necessarily correlate with a productive endocytosis pathway and hence needs further clarification (Table 2).

Some evidences have also revealed the role of fusion of KSHV envelope glycoproteins with target cell membranes (Akula et al., 2001a; Wang et al., 2001, 2003; Naranatt et al., 2002). The minimal fusion machinery of KSHV probably comprises of $\mathrm{gB}, \mathrm{gH}$, and gL since anti-gB, gH, gL, and gpK8.1A antibodies neutralize $\mathrm{KSHV}$ infection without affecting virus binding to the target cells (Akula et al., 2001a; Wang et al., 2001, 2003; Naranatt et al., 2002). However the mechanism of neutralization is not known. Multiple mechanisms could be possible depending upon the glycoprotein and the targeted region of the glycoproteins. For example, neutralization could be due to: (a) interference in the interaction of KSHV glycoproteins with integrins and other receptors; (b) interference at the activation of a subset of signal molecules that are essential for the $\mathrm{c}-\mathrm{Cbl}$ mediated receptor translocation, ubiquitination, bleb formation, and macropinocytosis; and (c) interference of fusion of viral envelope with the endosomal membrane. Further studies need to be carried out to precisely define the mechanism behind the neutralization by these antibodies. Nevertheless, these studies suggested that these glycoproteins play critical roles in the entry process after attachment has occurred, possibly as a result of interaction with additional cell surface molecules.

\section{POST ENTRY STEPS: KSHV TRAFFICKING AND PRODUCTIVE INFECTION OF KSHV}

Like most herpesviruses, KSHV replicates in the nucleus of infected cells. Therefore, to reach the nucleus KSHV capsids traffic through the crowded cytosol before releasing viral DNA into the nucleus (Lyman and Enquist, 2009). Delivery of KSHV DNA into the infected cell nucleus reached a peak by 90 min p.i. suggesting the rapidness of the host cellular trafficking utilized by the virus (Naranatt et al., 2003, 2005; Krishnan et al., 2004; Veettil et al., 2006; Raghu et al., 2009).

Productive infection comprised of KSHV internalized by macropinocytosis in endothelial cells or by clathrin-mediated endocytosis in fibroblast cells utilize the extensive microtubule (MT) network to traffic through the cytosol, tightly regulated by Rho-GTPases. Microtubule bundles colocalized with KSHV capsids and this colocalization was abolished by the microtubule destabilizing agent, nocodazole, and PI3-K inhibitor affecting the Rho-GTPases (Naranatt et al., 2003, 2004). Depolymerization of microtubules did not affect KSHV binding and internalization, but nuclear delivery of viral DNA and infection in HFF, HMVEC-d, and HUVEC cells was inhibited (Naranatt et al., 2005; Veettil et al., 2006; Raghu et al., 2009). Inhibition of Rho-GTPase activities by CdTxB abolished microtubular acetylation and subsequently the delivery of viral DNA to the nucleus. Conversely, activation of Rho-GTPases by Escherichia coli cytotoxic necrotizing factor significantly increased the intracellular trafficking and delivery of viral capsids to the nucleus. Similarly, nuclear delivery of viral DNA was increased in cells expressing a constitutively active RhoA mutant and decreased in cells expressing a dominant-negative mutant of RhoA (Naranatt et al., 2005). Taken together, these studies indicate that KSHV induces Rho-GTPases, modulates stabilization of microtubules and promotes the rapid trafficking of viral capsids toward the nucleus (Table 3 ).

Kaposi's sarcoma associated herpesvirus utilizes ATP dependant directional transport along microtubules governed by motor proteins. Dynein motor proteins responsible for minus-end transport from the periphery to the cell center actively carry KSHV to the vicinity of the nuclear membrane. Inhibition of dynein activity by sodium orthovanadate significantly reduced the infection and delivery of KSHV DNA into the nucleus (Naranatt et al., 2005). These studies demonstrate that KSHV movement across the cytoplasm to reach the nucleus is a series of well-orchestrated phenomenon probably involving viral proteins and host proteins. Further studies are essential to define this process and the mediating viral and host proteins.

Cross talk between ECM and integrins activates FAK, which initiates a cascade of intracellular signals that eventually activate the mitogen activated protein kinase (MAPK) pathways. As early as 5 min p.i., KSHV activates MEK (MAPK/ERK kinase) and extracellular-signal-regulated kinase (ERK; Naranatt et al., 2005). Soluble KSHV gpK8.1A, but not gB, induced MEK-mediated ERK1/2 phosphorylation as early as $5 \mathrm{~min}$ p.i., and ERK1/2 phosphorylation facilitated the establishment of KSHV infection in HFF and HMVEC-d cells (Sharma-Walia et al., 2005). PI3-K and protein kinase $\mathrm{C}-\zeta$ (PKC-zeta) are recruited as upstream mediators of the KSHV ERK pathway and inhibitors specific for PI3-K, PKC$\zeta$, MEK, and ERK significantly reduce virus infectivity without affecting virus binding to the target cells (Table 3 ).

\section{Table 3 | Host molecules and their roles in KSHV entry/infection in HMVEC-d and HFF cells.}

\begin{tabular}{ll}
\hline Molecules & Function in KSHV biology \\
\hline Heparan sulfate $(H S)$ & Binding receptor \\
Integrins $(\alpha 3 \beta 1, \alpha \vee \beta 3, \alpha \vee \beta 5)$ & Entry receptors \\
xCT/CD98 & Entry (fusion) receptor \\
FAK & Initiate endocytosis \\
Src & Initiate endocytosis \\
PI3-K, RhoA, Dia-2, Ezrin & Entry, actin modulation, endocytosis, and \\
& nuclear trafficking \\
C-Cbl & KSHV-receptor translocations into LR and \\
& adaptor for macropinocytosis in HMVEC-d \\
& cells
\end{tabular}

ERK and NF-кB Viral and host gene expression 
Rapid activation of $\mathrm{NF}-\kappa \mathrm{B}$ as early as $5-15 \mathrm{~min}$ p.i. led to the translocation of $\mathrm{p} 65-\mathrm{NF}-\kappa \mathrm{B}$ into the nucleus (Sadagopan et al., 2007). KSHV incubated with heparin significantly reduced $\mathrm{NF}-\kappa \mathrm{B}$ activation. During the observed $72 \mathrm{~h}$ periods of in vitro KSHV latency, a sustained moderate level of NF- $\kappa \mathrm{B}$ induction was observed, and inhibition of IКB phosphorylation by Bay117082 drastically reduced this activation (Sadagopan et al., 2007). In contrast, high levels of ERK1/2 activation during the earlier time points and a moderate level of activation at later time points were observed. The p38-MAP kinase was activated only during the later time points, and AKT was activated at lower levels in a cyclic manner. Studies with UV-KSHV suggested a role for virus entry stages in NF- $\kappa B$ induction and requirement of KSHV viral gene expression for sustained induction. Though inhibition of NF- $\kappa \mathrm{B}$ did not have any effect on KSHV entry into cells, expression of viral latent ORF 73 and lytic ORF 50 genes was significantly reduced. Several transcription factors were activated during KSHV infection, and inhibition of NF- $\kappa$ B significantly affected the activation of Jun D, Jun B, phospho-c-Jun, cFos, and FosB factors. These results suggested that during in vitro infection, KSHV induces sustained levels of NF- $\kappa \mathrm{B}$ to regulate viral genes thus possibly regulating the establishment of latent infection.

Productive KSHV primary infection in adherent cells involves an initial lytic phase ( $2 \mathrm{~h}$ p.i.) succeeded by a latent phase $(24 \mathrm{~h}$ p.i.) with a decline of lytic phase (Krishnan et al., 2004). A report by Yoo et al. (2005) suggests that KSHV infection is permissive in HUVECs at early time points with an initial production of infectious virus particles (lytic cycle), while the surviving cells later enter a latent phase with spontaneous lytic replication. However, the percentage of cells that were infected, cells that entered into lytic cycle and cells that entered latency were not determined in these studies. Hence, these studies need to be reexamined.

\section{REFERENCES}

Akula, S. M., Naranatt, P. P., Walia, N. S., Wang, F. Z., Fegley, B., and Chandran, B. (2003). Kaposi's sarcomaassociated herpesvirus (human herpesvirus 8) infection of human fibroblast cells occurs through endocytosis. J. Virol. 77, 7978-7990.

Akula, S. M., Pramod, N. P., Wang, F. Z., and Chandran, B. (2001a). Human herpesvirus 8 envelopeassociated glycoprotein B interacts with heparan sulfate-like moieties. Virology 284, 235-249.

Akula, S. M., Wang, F. Z., Vieira, J., and Chandran, B. (2001b). Human herpesvirus 8 interaction with target cells involves heparan sulfate. Virology 282, 245-255.

Akula, S. M., Pramod, N. P., Wang, F. Z., and Chandran, B. (2002). Integrin alpha3betal (CD 49c/29) is a cellular receptor for Kaposi's sarcoma-associated herpesvirus (KSHV/HHV-8) entry into the target cells. Cell 108, 407-419.

Baghian, A., Luftig, M., Black, J. B., Meng, Y. X., Pau, C. P., Voss, T., Pellett, P. E., and Kousoulas, K. G.

\section{PERSPECTIVES AND FUTURE DIRECTIONS}

Kaposi's sarcoma associated herpesvirus exemplifies an excellent model for viruses requiring multiple cellular molecules to enter target cells. Although, integrins and associated signaling, adaptor molecules and a host of pre-existing signaling molecules have been identified to play a crucial role in KSHV entry and infection, the nature and mechanism by which the virus is able to successfully utilize the sequential series of host signaling still remains a mystery. Moreover, there is a difference in the entry pathways of KSHV in infectable cell types, making this interesting story more complicated. What is clear from studies so far is that KSHV overcomes cellular barriers making conditions conducive to infection by utilizing more than one endocytic mechanism. However, further studies need to be performed to ensure whether differential activation of signal responses by KSHV coupled to different endosomal vesicles accounts for the varied modes of entry. Moreover, whether KSHV utilizes more than one E3-ubiquitin ligase and their modulation by viral induced cellular targets remains to be explored. Another interesting future study involves the recognition of KSHV in the endosomal vesicles that direct productive trafficking of virus away from the lysosomal compartments. Cellular signaling targets of KSHV are broad and hence critical molecules regulating such multiple pathways need to be targeted for therapeutics. These studies will shed valuable insights into the molecular mechanisms of cytosolic entry of KSHV.

\section{ACKNOWLEDGMENTS}

This study was supported in part by Public Health Service grants (NIH) CA 075911 and CA 168472, and the Rosalind Franklin University of Medicine and Science H. M. Bligh Cancer Research Fund to Bala Chandran. We thank Keith Philibert for critically reading the manuscript.

infects endothelial and spindle cells. Nat. Med. 1, 1274-1278.

Calderwood, D. A., Shattil, S. J., and Ginsberg, M. H. (2000). Integrins and actin filaments: reciprocal regulation of adhesion and signaling. $J$. Biol. Chem. 275, 22607-22610.

Cesarman, E., Nador, R. G., Bai, F., Bohenzky, R. A., Russo, J. J., Moore, P. S., Chang, Y., and Knowles, D. M. (1996). Kaposi's sarcomaassociated herpesvirus contains $G$ protein-coupled receptor and cyclin D homologs which are expressed in Kaposi's sarcoma and malignant lymphoma. J. Virol. 70, 8218-8223.

Chakraborty, S., Valiyaveettil, M., Sadagopan, S., Paudel, N., and Chandran, B. (2011). c-Cblmediated selective virus-receptor translocations into lipid rafts regulate productive Kaposi's sarcoma-associated herpesvirus infection in endothelial cells. $J$. Virol. 85, 12410-12430.

Dourmishev, L. A., Dourmishev, A. L., Palmeri, D., Schwartz, R. A., and Lukac, D. M. (2003). Molecular genetics of Kaposi's sarcomaassociated herpesvirus (human herpesvirus-8) epidemiology and pathogenesis. Microbiol. Mol. Biol. Rev. 67, 175-212.

Dupin, N., Fisher, C., Kellam, P., Ariad, S., Tulliez, M., Franck, N., van Marck, E., Salmon, D., Gorin, I., Escande, J. P., Weiss, R. A., Alitalo, K., and Boshoff, C. (1999). Distribution of human herpesvirus8 latently infected cells in Kaposi's sarcoma, multicentric Castleman's disease, and primary effusion lymphoma. Proc. Natl. Acad. Sci. U.S.A. 96, 4546-4551.

Fenczik, C. A., Zent, R., Dellos, M., Calderwood, D. A., Satriano, J., Kelly, C., and Ginsberg, M. H. (2001). Distinct domains of $\mathrm{CD} 98 \mathrm{hc}$ regulate integrins and amino acid transport. J. Biol. Chem. 276, 8746-8752.

Feral, C. C., Zijlstra, A., Tkachenko, E., Prager, G., Gardel, M. L., Slepak, M., and Ginsberg, M. H. (2007). CD98hc (SLC3A2) participates in fibronectin matrix assembly by mediating integrin signaling. $J$. Cell Biol. 178, 701-711. 
Ganem, D. (2007a). "Kaposi's sarcoma associated herpesvirus," in Fields Virology, 5th Edn, Vol. 2, eds D. M. Knipe and P. M. Howley (Philadelphia, PA: Lippincott Williams \& Wilkins), 2875-2888.

Ganem, D. (2007b). KSHV-Induced Oncogenesis. Human Herpesviruses Biology, Therapy, and Immunoprophylaxis, Chap. 56. Cambridge: Cambridge University Press, 1007-1028.

Garrigues, H. J., Rubinchikova, Y. E., Dipersio, C. M., and Rose, T. M. (2008). Integrin alphaVbeta3 Binds to the RGD motif of glycoprotein B of Kaposi's sarcoma-associated herpesvirus and functions as an RGDdependent entry receptor. J. Virol. 82, 1570-1580.

Geijtenbeek, T. B., Van Vliet, S. J., Koppel, E. A., Sanchez-Hernandez, M., Vandenbroucke-Grauls, C. M., Appelmelk, B., and Van Kooyk, Y. (2003). Mycobacteria target DCSIGN to suppress dendritic cell function. J. Exp. Med. 197, 7-17.

Giancotti, F. G. (2000). Complexity and specificity of integrin signalling. Nat. Cell Biol. 2, E13-E14.

Giancotti, F. G. (2003). A structural view of integrin activation and signaling. Dev. Cell 4, 149-151.

Greene, W., and Gao, S. J. (2009). Actin dynamics regulate multiple endosomal steps during Kaposi's sarcoma-associated herpesvirus entry and trafficking in endothelial cells. PLoS Pathog. 5, e1000512. doi:10.1371/journal.ppat.1000512

Grundhoff, A., and Ganem, D. (2004). Inefficient establishment of KSHV latency suggests an additional role for continued lytic replication in Kaposi sarcoma pathogenesis. J. Clin. Invest. 113, 124-136.

Hahn, A., Birkmann, A., Wies, E., Dorer, D., Mahr, K., Sturzl, M., Titgemeyer, F., and Neipel, F. (2009). Kaposi's sarcoma-associated herpesvirus gH/gL: glycoprotein export and interaction with cellular receptors. J. Virol. 83, 396-407.

Hall, A., and Nobes, C. D. (2000). Rho GTPases: molecular switches that control the organization and dynamics of the actin cytoskeleton. Philos. Trans. R. Soc. Lond. B Biol. Sci. 355, 965-970.

Inoue, N., Winter, J., Lal, R. B., Offermann, M. K., and Koyano, S. (2003). Characterization of entry mechanisms of human herpesvirus 8 by using an Rta-dependent reporter cell line. J. Virol. 77, 8147-8152.

Ishizaki, T., Morishima, Y., Okamoto, M., Furuyashiki, T., Kato, T., and Narumiya, S. (2001). Coordination of microtubules and the actin cytoskeleton by the Rho effector mDia1. Nat. Cell Biol. 3, 8-14.

Jarousse, N., Chandran, B., and Coscoy, L. (2008). Lack of heparan sulfate expression in B-cell lines: implications for Kaposi's sarcomaassociated herpesvirus and murine gammaherpesvirus 68 infections. J. Virol. 82, 12591-12597.

Kabir-Salmani, M., Fukuda, M. N., Kanai-Azuma, M., Ahmed, N., Shiokawa, S., Akimoto, Y., Sakai, K., Nagamori, S., Kanai, Y., Sugihara, K., and Iwashita, M. (2008). The membrane-spanning domain of CD98 heavy chain promotes alpha(v)beta3 integrin signals in human extravillous trophoblasts. Mol. Endocrinol. 22, 707-715.

Kaleeba, J. A., and Berger, E. A. (2006). Broad target cell selectivity of Kaposi's sarcoma-associated herpesvirus glycoprotein-mediated cell fusion and virion entry. Virology 354, 7-14.

Kerur, N., Veettil, M. V., Sharma-Walia, N., Sadagopan, S., Bottero, V., Paul, A. G., and Chandran, B. (2010). Characterization of entry and infection of monocytic THP-1 cells by Kaposi's sarcoma associated herpesvirus (KSHV): role of heparan sulfate, DC-SIGN, integrins and signaling. Virology 406, 103-116.

Koyano, S., Mar, E. C., Stamey, F. R., and Inoue, N. (2003). Glycoproteins M and $\mathrm{N}$ of human herpesvirus 8 form a complex and inhibit cell fusion. $J$. Gen. Virol. 84, 1485-1491.

Krishnan, H. H., Naranatt, P. P., Smith, M. S., Zeng, L., Bloomer, C., and Chandran, B. (2004). Concurrent expression of latent and a limited number of lytic genes with immune modulation and antiapoptotic function by Kaposi's sarcoma-associated herpesvirus early during infection of primary endothelial and fibroblast cells and subsequent decline of lytic gene expression. J. Virol. 78, 3601-3620.

Krishnan, H. H., Sharma-Walia, N., Streblow, D. N., Naranatt, P. P., and Chandran, B. (2006). Focal adhesion kinase is critical for entry of Kaposi's sarcoma-associated herpesvirus into target cells. J. Virol. 80, 1167-1180.

Krishnan, H. H., Sharma-Walia, N., Zeng, L., Gao, S. J., and Chandran, B. (2005). Envelope glycoprotein gB of Kaposi's sarcomaassociated herpesvirus is essential for egress from infected cells. J. Virol. 79, 10952-10967.

Levkowitz, G., Waterman, H., Zamir, E., Kam, Z., Oved, S., Langdon,
W. Y., Beguinot, L., Geiger, B., and Yarden, Y. (1998). c-Cbl/Sli-1 regulates endocytic sorting and ubiquitination of the epidermal growth factor receptor. Genes Dev. 12 , 3663-3674.

Liao, W., Tang, Y., Kuo, Y. L., Liu, B. Y., Xu, C. J., and Giam, C. Z. (2003). Kaposi's sarcoma-associated herpesvirus/human herpesvirus 8 transcriptional activator Rta is an oligomeric DNA-binding protein that interacts with tandem arrays of phased A/T-trinucleotide motifs. J. Virol. 77, 9399-9411.

Lozach, P. Y., Kuhbacher, A., Meier, R., Mancini, R., Bitto, D., Bouloy, M. and Helenius, A. (2011). DC-SIGN as a receptor for phleboviruses. Cell Host Microbe 10, 75-88.

Lyman, M. G., and Enquist, L. W. (2009). Herpesvirus interactions with the host cytoskeleton. J. Virol. 83, 2058-2066.

Mark, L., Lee, W. H., Spiller, O. B., Villoutreix, B. O., and Blom, A. M. (2006). The Kaposi's sarcomaassociated herpesvirus complement control protein (KCP) binds to heparin and cell surfaces via positively charged amino acids in CCP12. Mol. Immunol. 43, 1665-1675.

Marsh, M., and Helenius, A. (2006). Virus entry: open sesame. Cell 124, 729-740.

Mercer, J., and Helenius, A. (2008). Vaccinia virus uses macropinocytosis and apoptotic mimicry to enter host cells. Science 320, 531-535.

Naranatt, P. P., Akula, S. M., and Chandran, B. (2002). Characterization of gamma2-human herpesvirus-8 glycoproteins gH and gL. Arch. Virol. 147, 1349-1370.

Naranatt, P. P., Akula, S. M., Zien, C. A., Krishnan, H. H., and Chandran, B. (2003). Kaposi's sarcomaassociated herpesvirus induces the phosphatidylinositol 3-kinase-PKCzeta-MEK-ERK signaling pathway in target cells early during infection: implications for infectivity. J. Virol. 77, 1524-1539.

Naranatt, P. P., Krishnan, H. H., Smith, M. S., and Chandran, B. (2005). Kaposi's sarcomaassociated herpesvirus modulates microtubule dynamics via RhoAGTP-diaphanous 2 signaling and utilizes the dynein motors to deliver its DNA to the nucleus. J. Virol. 79, 1191-1206.

Naranatt, P. P., Krishnan, H. H., Svojanovsky, S. R., Bloomer, C., Mathur, S., and Chandran, B. (2004). Host gene induction and transcriptional reprogramming in Kaposi's sarcoma-associated herpesvirus
(KSHV/HHV-8)-infected endothelial, fibroblast, and B cells: insights into modulation events early during infection. Cancer Res. 64, 72-84.

Neipel, F., Albrecht, J. C., and Fleckenstein, B. (1997). Cell-homologous genes in the Kaposi's sarcomaassociated rhadinovirus human herpesvirus 8: determinants of its pathogenicity? J. Virol. 71, 4187-4192.

Palazzo, A. F., Cook, T. A., Alberts, A. S., and Gundersen, G. G. (2001). mDia mediates Rho-regulated formation and orientation of stable microtubules. Nat. Cell Biol. 3, 723-729.

Parravicini, C., Chandran, B., Corbellino, M., Berti, E., Paulli, M., Moore, P. S., and Chang, Y. (2000). Differential viral protein expression in Kaposi's sarcomaassociated herpesvirus-infected diseases: Kaposi's sarcoma, primary effusion lymphoma, and multicentric Castleman's disease. Am. J. Pathol. 156, 743-749.

Raghu, H., Sharma-Walia, N., Veettil, M. V., Sadagopan, S., Caballero, A., Sivakumar, R., Varga, L., Bottero, V., and Chandran, B. (2007). Lipid rafts of primary endothelial cells are essential for Kaposi's sarcomaassociated herpesvirus/human herpesvirus 8-induced phosphatidylinositol 3-kinase and RhoA-GTPases critical for microtubule dynamics and nuclear delivery of viral DNA but dispensable for binding and entry. J. Virol. 81, 7941-7959.

Raghu, H., Sharma-Walia, N., Veettil, M. V., Sadagopan, S., and Chandran, B. (2009). Kaposi's sarcomaassociated herpesvirus utilizes an actin polymerization-dependent macropinocytic pathway to enter human dermal microvascular endothelial and human umbilical vein endothelial cells. J. Virol. 83, 4895-4911.

Rappocciolo, G., Hensler, H. R., Jais, M., Reinhart, T. A., Pegu, A., Jenkins, F. J., and Rinaldo, C. R. (2008). Human herpesvirus 8 infects and replicates in primary cultures of activated $\mathrm{B}$ lymphocytes through DC-SIGN. J. Virol. 82, 4793-4806.

Rappocciolo, G., Jenkins, F. J., Hensler, H. R., Piazza, P., Jais, M., Borowski, L., Watkins, S. C., and Rinaldo, C. R. Jr. (2006). DC-SIGN is a receptor for human herpesvirus 8 on dendritic cells and macrophages. J. Immunol. 176, 1741-1749.

Riveline, D., Zamir, E., Balaban, N. Q., Schwarz, U. S., Ishizaki, T., Narumiya, S., Kam, Z., Geiger, B., and Bershadsky, A. D. (2001). Focal contacts as mechanosensors: externally applied local mechanical force 
induces growth of focal contacts by an mDial-dependent and ROCKindependent mechanism. J. Cell Biol. 153, 1175-1186.

Sadagopan, S., Sharma-Walia, N., Veettil, M. V., Raghu, H., Sivakumar, R., Bottero, V., and Chandran, B. (2007). Kaposi's sarcomaassociated herpesvirus induces sustained NF-kappaB activation during de novo infection of primary human dermal microvascular endothelial cells that is essential for viral gene expression. J. Virol. 81, 3949-3968.

Sastry, S. K., and Burridge, K. (2000). Focal adhesions: a nexus for intracellular signaling and cytoskeletal dynamics. Exp. Cell Res. 261, 25-36.

Schmidt, M. H., and Dikic, I. (2005). The $\mathrm{Cbl}$ interactome and its functions. Nat. Rev. Mol. Cell Biol. 6, 907-918.

Sharma-Walia, N., Krishnan, H. H., Naranatt, P. P., Zeng, L., Smith, M. S., and Chandran, B. (2005). ERK1/2 and MEK1/2 induced by Kaposi's sarcoma-associated herpesvirus (human herpesvirus 8) early during infection of target cells are essential for expression of viral genes and for establishment of infection. J. Virol. 79, 10308-10329.

Sharma-Walia, N., Naranatt, P. P., Krishnan, H. H., Zeng, L., and Chandran,
B. (2004). Kaposi's sarcomaassociated herpesvirus/human herpesvirus 8 envelope glycoprotein $\mathrm{gB}$ induces the integrin-dependent focal adhesion kinase-Srcphosphatidylinositol 3-kinase-rho GTPase signal pathways and cytoskeletal rearrangements. J. Virol. 78, 4207-4223.

Simmons, G. (2001). Lipid rafts and signal transduction. Nat. Rev. Mol. Cell Biol. 2, 216.

Thien, C. B., and Langdon, W. Y. (2001). Cbl: many adaptations to regulate protein tyrosine kinases. Nat. Rev. Mol. Cell Biol. 2, 294-307.

Valiya Veettil, M., Sadagopan, S., Kerur, N., Chakraborty, S., and Chandran, B. (2010). Interaction of c-Cbl with myosin IIA regulates Bleb associated macropinocytosis of Kaposi's sarcoma-associated herpesvirus. PLoS Pathog. 6, e1001238. doi:10.1371/journal.ppat. 1001238

Veettil, M. V., Sadagopan, S., SharmaWalia, N., Wang, F. Z., Raghu, H., Varga, L., and Chandran, B. (2008). Kaposi's sarcoma-associated herpesvirus forms a multimolecular complex of integrins (alphaVbeta5, alphaVbeta3, and alpha3beta1) and CD98-xCT during infection of human dermal microvascular endothelial cells, and CD98-xCT is essential for the postentry stage of infection. J. Virol. 82, 12126-12144.
Veettil, M. V., Sharma-Walia, N., Sadagopan, S., Raghu, H., Sivakumar, R., Naranatt, P. P., and Chandran, B. (2006). RhoA-GTPase facilitates entry of Kaposi's sarcomaassociated herpesvirus into adherent target cells in a Src-dependent manner. J. Virol. 80, 11432-11446.

Wang, F. Z., Akula, S. M., Pramod, N. P., Zeng, L., and Chandran, B. (2001). Human herpesvirus 8 envelope glycoprotein K8.1A interaction with the target cells involves heparan sulfate. J. Virol. 75, 7517-7527.

Wang, F. Z., Akula, S. M., SharmaWalia, N., Zeng, L., and Chandran, B. (2003). Human herpesvirus 8 envelope glycoprotein B mediates cell adhesion via its RGD sequence. $J$. Virol. 77, 3131-3147.

Yoo, S. M., Zhou, F. C., Ye, F. C., Pan, H. Y., and Gao, S. J. (2005). Early and sustained expression of latent and host modulating genes in coordinated transcriptional program of KSHV productive primary infection of human primary endothelial cells. Virology 343, 47-64.

Zhu, L., Puri, V., and Chandran, B. (1999a). Characterization of human herpesvirus-8 K8.1A/B glycoproteins by monoclonal antibodies. Virology 262, 237-249.

Zhu, L., Wang, R., Sweat, A., Goldstein, E., Horvat, R., and Chandran, B. (1999b). Comparison of human sera reactivities in immunoblots with recombinant human herpesvirus (HHV)-8 proteins associated with the latent (ORF73) and lytic (ORFs $65, \mathrm{~K} 8.1 \mathrm{~A}$, and $\mathrm{K} 8.1 \mathrm{~B}$ ) replicative cycles and in immunofluorescence assays with HHV-8-infected BCBL-1 cells. Virology 256, 381-392.

Conflict of Interest Statement: The authors declare that the research was conducted in the absence of any commercial or financial relationships that could be construed as a potential conflict of interest.

Received: 19 November 2011; paper pending published: 11 December 2011; accepted: 05 January 2012; published online: 20 January 2012.

Citation: Chakraborty S, Veettil MV and Chandran B (2012) Kaposi's sarcoma associated herpesvirus entry into target cells. Front. Microbio. 3:6. doi: 10.3389/fmicb.2012.00006

This article was submitted to Frontiers in Virology, a specialty of Frontiers in Microbiology.

Copyright (C) 2012 Chakraborty, Veettil and Chandran. This is an open-access article distributed under the terms of the Creative Commons Attribution Non Commercial License, which permits noncommercial use, distribution, and reproduction in other forums, provided the original authors and source are credited. 